\title{
How Cutting-Edge Atomic Resolution Microscopy Can Help to Solve Some of the World's Energy Problems
}

\author{
Colin Humphreys \\ Department of Materials Science and Metallurgy, University of Cambridge, Cambridge, CB3 OFS, UK
}

The world's energy demands are growing more steeply now than at any time in the last 200 years (when detailed records began). They are being driven by an increase in the world's population and by increased usage of cars, planes, mobile phones, etc. Fortunately the world's energy supply is greater than had been expected because of the recent exploitation of shale gas and oil. However, for the world as a whole there is an increasing gap between energy demand and supply.

The most effective way to close the gap between energy demand and supply is improved energy efficiency. The US Department of Energy has surveyed all the ways in which electricity is used and concluded that the area with the largest energy savings potential is LED lighting. This talk will show how cutting edge microscopy has been essential to develop lowcost high-efficiency LED lighting based on gallium nitride (GaN). GaN is also important for next-generation high-efficiency power electronic devices. Potentially about $25 \%$ of our electricity consumption can be saved using such energy efficient LEDs and power electronic devices. GaN is also important for next-generation solar cells. The ternary alloy InGaN has a bandgap that covers most of the solar spectrum (bandgap of $\mathrm{InN}$ is $0.7 \mathrm{eV}$ and of $\mathrm{GaN}$ is $3.4 \mathrm{eV}$ ). Multi-junction solar cells made from InGaN have a theoretical efficiency of over $70 \%$ and hence show huge promise for renewable solar energy.

The light-emitting layers in GaN LEDs are quantum wells (QWs) of InGaN sandwiched between GaN barriers. The QWs emit blue light and a covering phosphor converts this to white. Bright emission is obtained for dislocation densities as high as $10^{9} \mathrm{~cm}^{-2}$, while for all other light-emitting semiconductors the dislocation density has to be less than $10^{3} \mathrm{~cm}^{-2}$, or the dislocations, as non-radiative recombination centres, quench the light emission. It was thought that nm-sized In-rich clusters in the QWs localised the carriers (electrons and holes) and prevented then from diffusing to the dislocations. However, such clusters, observed in electron microscopy, were shown to be an artefact of electron-beam damage [1], changing the accepted understanding of why GaN LEDs are so efficient with high dislocation densities.

So what mechanism is localising the carriers in the InGaN quantum wells? Aberration corrected electron microscopy was required to show that the quantum wells contained monolayer-height surface steps, and theoretical calculations showed that these would localise the electrons [2]. By varying the growth conditions, the density of monolayer-height surface steps

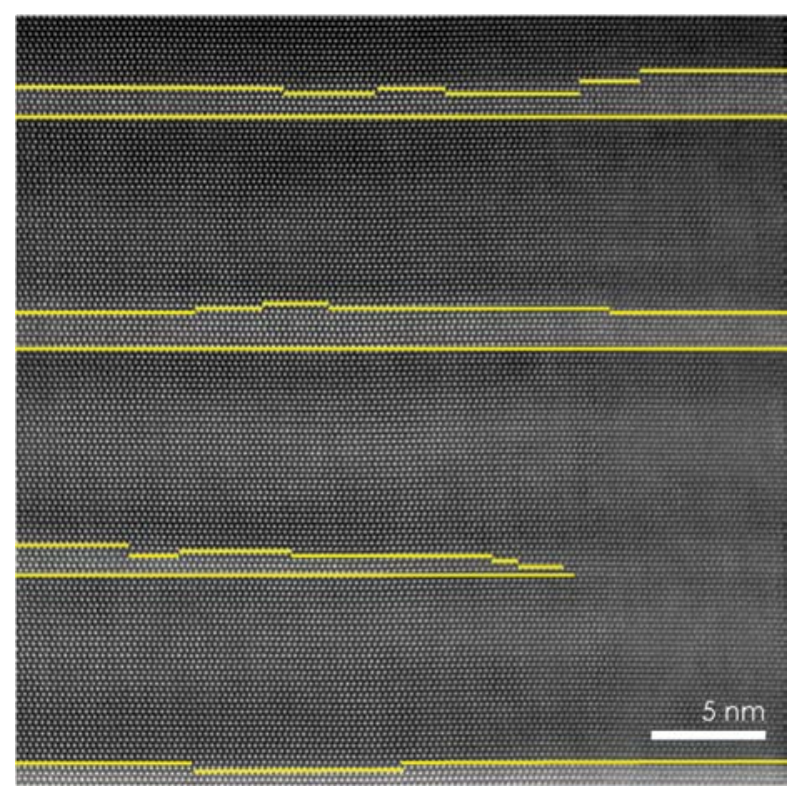

Figure 1. InGaN QWs showing monolayer interface steps. Cs corrected Titan 80-300 HAADF image courtesy of SL Sahonta. can be controlled (Figure 1). Since electron microscopy showed that InGaN QWs do not contain Indium-rich clusters, InGaN is probably a random alloy. Another form of microscopy, atom probe tomography (APT), was used to confirm this [3] (Figure 2). Data from electron microscopy and APT were then used in quantum mechanical calculations to show that the holes were strongly localised by random indium fluctuations in the InGaN quantum wells [4]. So microscopy has been essential in understanding the fundamental science of GaN-based LEDs.

Microscopy then became essential in the technological development of GaN-based LEDs. The main factor preventing the widespread use of LED lighting in our homes and offices is cost. A $60 \mathrm{~W}$ equivalent replacement LED bulb currently costs about $\$ 15$, which most people will not spend. GaN LEDs are currently expensive because they are grown on small diameter (2-inch or 4-inch) sapphire or $\mathrm{SiC}$ substrates. Substantial cost reductions are possible if they are grown on 6-inch $\mathrm{Si}$ substrates. However $\mathrm{GaN}$ cannot be grown directly on $\mathrm{Si}$ because it reacts to form a Ga-Si alloy, so an AlN nucleation layer is first grown on the Si. The quality of the AlN/Si interface turns out to be very important and aberration corrected microscopy was again essential to show the existence of a $2 \mathrm{~nm}$ thick amorphous layer at the interface (Figure 3). Electron energy loss elemental images were 


\section{Plenary Special Lectures}

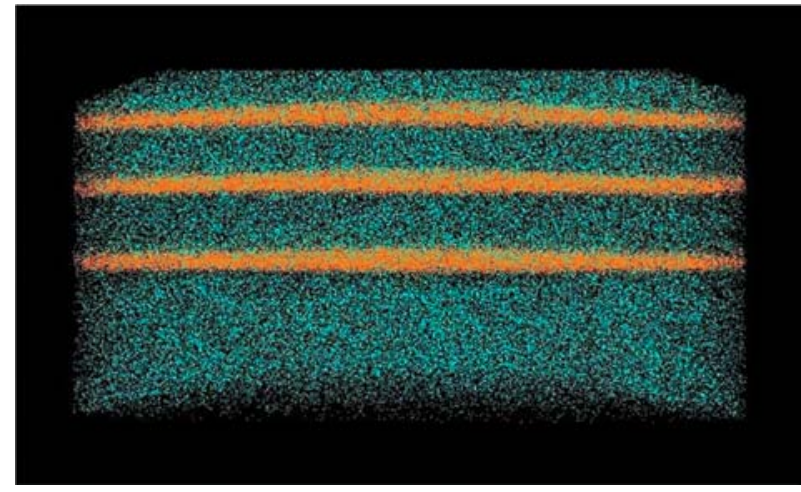

Figure 2. APT image of InGaN quantum wells and GaN barriers. In atoms orange, $\mathrm{Ga}$ atoms green.

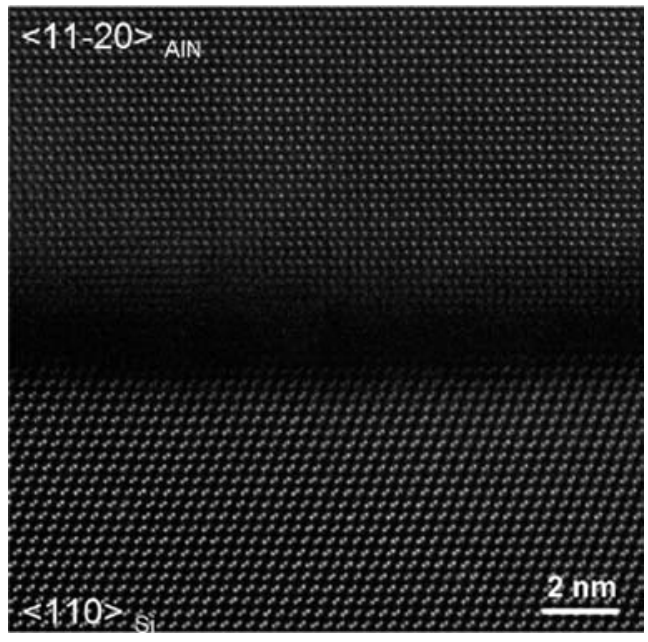

Figure 3. AIN on $\mathrm{Si}$ showing amorphous $\mathrm{Si}_{x} \mathrm{~N}_{y}$ layer at the interface. Cs corrected Titan 80-300 HAADF image.

then used to show that this layer was $\mathrm{Si}_{\mathrm{x}} \mathrm{N}_{\mathrm{y}}$ [5]. Thus advanced electron microscopy techniques have been essential to understand the science of GaN LEDs and to develop the technology. If widely used, such LEDs will save 10-15\% of electricity.

Advanced electron microscopy is similarly proving invaluable in developing GaN-based power electronic devices. If widely used these would save a further $9 \%$ of electricity. We can also expect that electron microscopy will be important in the future development of InGaN solar cells, which have the potential to be the most efficient solar cells in the world. Thus electron microscopy has a vital role to play in solving the world's energy problems.

References:

[1] TM Smeeton et al, Appl. Phys. Lett., 83 (2003), 5419.

[2] DM Graham et al, J. Appl. Phys., 97 (2005) 103508.

[3] MA Galtrey et al, Appl. Phys. Lett., 90 (2007) 061903.

[4] D Watson-Parris et al, Phys. Rev. B, 83 (2011) 115321.

[5] G Radtke et al, Appl. Phys. Lett., 100 (2012) 011910.

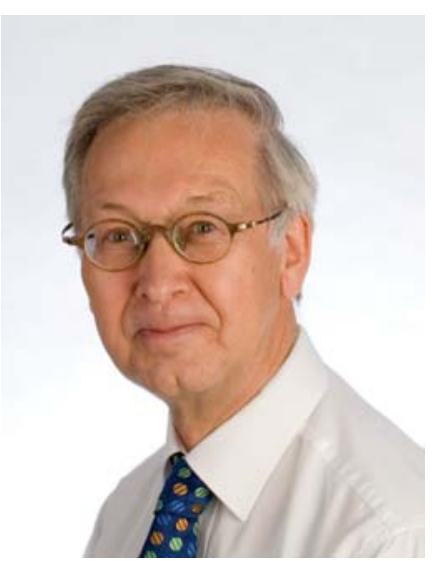

Colin Humphreys
Professor Humphreys is Director of Research in the Department of Materials Science and Metallurgy, University of Cambridge. He is also the Director of the Cambridge Centre for Gallium Nitride and the Rolls-Royce/Cambridge University Technology Partnership for Advanced Aerospace Materials. His early work in electron microscopy focused on using electron diffraction theory to interpret the images of defects in a wide range of materials and he also developed new electron microscope techniques. More recently he has used electron microscopy and atom probe tomography (APT) for the advanced characterisation of materials. In this talk he will show how atomic resolution electron microscopy and APT can be used to help to solve some of the world's energy problems and can also enable the commercial exploitation of materials. 
How Pioneering Natural Philosophers Observed the Microscopical World

Brian J Ford

Fellow, Cardiff University. Rothay House, Mayfield Road, Eastrea, Cambridge PE7 2AY

This research has determined how microscopy began. Until the mid-17th century, no investigator had glimpsed the complexity of living cells, or discerned the communities of microscopic organisms that are everywhere around us. Since that time, many scientific works and television programs have reported how difficult it was to make useful observations through the early microscopes. It has now been shown that this was not due to deficiencies in instruments. When used correctly they reveal extraordinary detail.

Science has lost the knowledge needed to tease the best images from these remarkable, pioneering instruments. When used correctly, the earliest microscopes are now shown to reveal a remarkable degree of resolution [1]. In the four examples below, original ancient lenses have been used. Figures $1 \& 2$ were taken with the Leeuwenhoek single lens microscope from Utrecht University (circa 1690); figures $3 \& 4$ are taken with Robert Brown's microscope (dating from 1827).

Video allows us to savor the appearance of specimens in real time, and innovative techniques have now been devised that allow us to reprise the way in which specimens appeared to the pioneering investigators. The initial results were presented at the Royal Society, London, in 2010 [2].



Figure 1. One of Leeuwenhoek's original microscopes is now preserved at Utrecht. This is the conventional view of a human blood specimen photographed through this instrument, dating from about 1690. No detail can be seen.

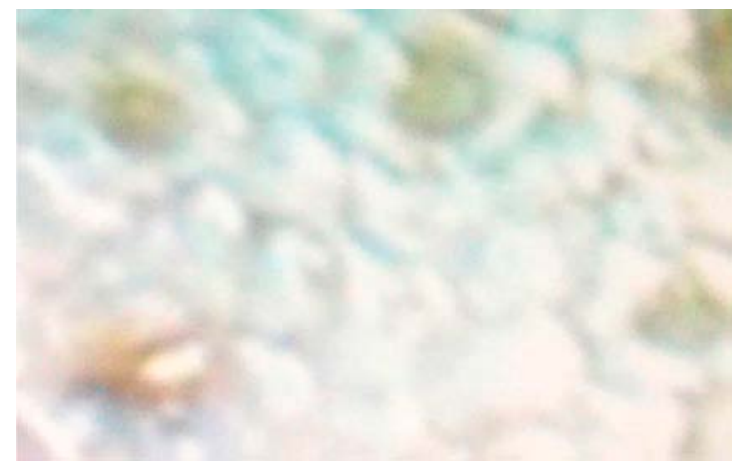

Figure 3. The recent BBC Television series entitled 'Cell' attempted to recreate the first observations that Robert Brown made of the cell nucleus in 1827. This is the result of their major six-figure budget: for all the effort and the BBC's technical expertise, little detail can be seen.

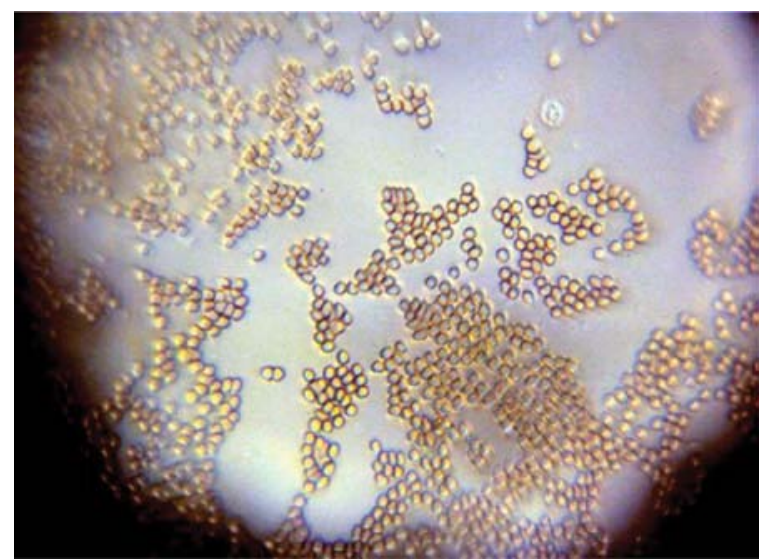

Figure 2. Used correctly, the microscope can generate this highly detailed image. There is a scattering of many red cells (erythrocytes) and, top right, a white cell (leucocyte) and its nucleus can be clearly discerned.

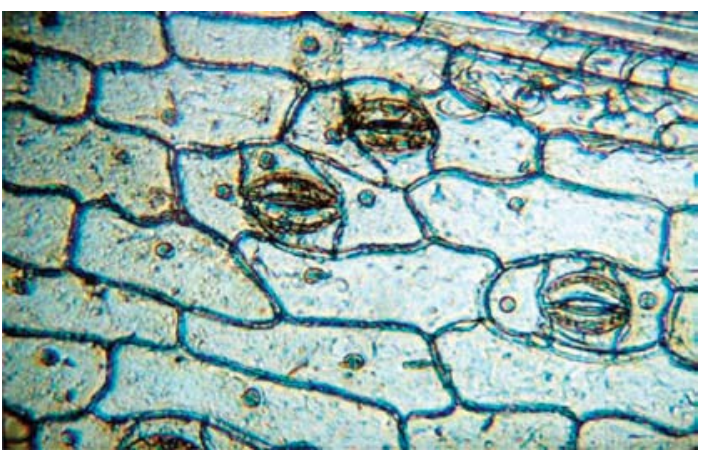

Figure 4. Using the same microscope, this is the remarkable amount of detail that Brown's microscope can reveal when set up correctly. Not only is the nucleus in each cell easily seen, but details of the stomata-the leaf's breathing pores - can also be resolved with ease.

\section{References:}

[1] B J Ford, Did physics matter to the pioneers of microscopy? Advances in Imaging and Electron Physics, 158: 27-87, 2009, New York: Academic Press.

[2] B J Ford, Revolutionary insights-the dawn of microscopical investigation, lunchtime lecture to Royal Society of London, October 29, 2010. 


\section{Plenary Special Lectures}

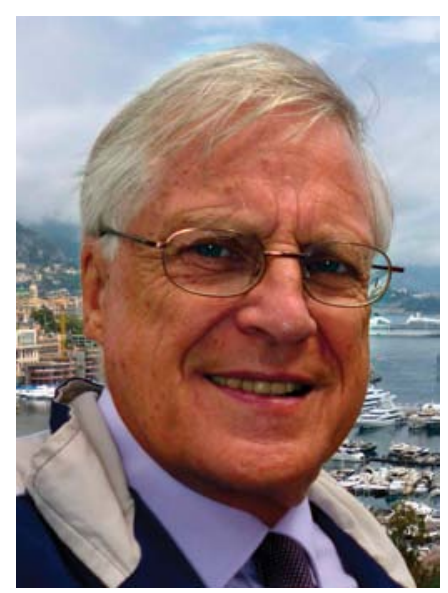

Brian J Ford
Brian J Ford is a prolific research scientist who launched major science programs for the BBC. His books pioneer new approaches in bringing science to the public. He was a NESTA Fellow 2004-2007, was presented with the inaugural Köhler medal in America for his work in microscopy, and has been nominated for the prestigious Faraday Medal of the Royal Society in London. He studied biology at Cardiff University, but left University to establish his own laboratory to work on a new interdisciplinary approach to scientific research. He was elected a Fellow of Cardiff University in 1986, has been a Member of the University Court since 1981, and has been President of the Association of Past Students. He has made unique contributions to science and is a world authority on the microscope (the subject of many of his best-selling books). He pioneered regular 'science reports' on television news, went on to introduce regular science to BBC radio and then launched his own programs. Ford's work has revolutionized many major areas of science. His BBC programs (Science Now, for instance) broke new ground in the public accessibility of science and were enthusiastically reviewed. His major TV series Food for Thought was in the network top ten within its second week of transmission; it was widely used as a teaching aid in schools. Another pioneering title was Microbe Power - Tomorrow's Revolution, for this painted a detailed portrait of the importance of microorganisms. Ford's microscope books, from Revealing Lens and Optical Microscope Manual to the more recent Single Lens and Leeuwenhoek Legacy have changed our understanding of the development of this important branch of science. In England he was highly influential as a Director of Mensa, and a member of Council and a Director of the National Science Centre project, whilst in the USA he has served on the boards of the McCrone Research Institute and the Van Leeuwenhoek Institute, has been appointed adviser to the National Science Foundation and is an Emeritus Fellow of the New York Academy of Science. At Cambridge University he is President of the Society for the Application of Research, and a member of Council of the Friends of the University Library. Among many posts, he held a Fellowship at the Open University 2001-2004; he is based at Gonville and Caius College, Cambridge University and is an honorary Member of Keynes College at the University of Kent. Recently he produced a microscope manual given to British schools as part of Science Year, and has published a book on GM crops that received enthusiastic reviews. Currently he has a 20,000 word chapter in press for a major physics textbook and has published several papers on the intelligence of living cells. 